\title{
USO DE ANTIDEPRESSIVOS NO MUNICÍPIO DE CAPITÃO LEÔNIDAS MARQUES - PR
}

\section{USE OF ANTIDEPRESSANTS IN CAPITÃO LEÔNIDAS MARQUES CITY - PR}

\author{
Luiz Felipe Perin*, Vagner Fagnani Linartevichi \\ Curso de Medicina do Centro Universitário Assis Gurgacz
}

*Autor correspondente: luizfp_95@hotmail.com, https://orcid.org/0000-0003-0331-2926

\section{RESUMO}

Esse é um estudo retrospectivo realizado na cidade de Capitão Leônidas Marques, Paraná, Brasil, com objetivo de delinear o perfil municipal de dispensação de antidepressivos no período de Janeiro de 2015 à Dezembro de 2017. Os dados foram obtidos a partir dos Relatórios Trimestrais (RT) de medicamentos psicotrópicos submetidos a controle especial pela Portaria 344/98 do MS, da rede privada (farmácias) e pública (SUS e Hospital), fornecidos pela vigilância sanitária. Os Antidepressivos correspondem a 55,3\% do total de psicotrópicos dispensados nesse período e o mais consumido foi Amitriptilina 25mg, sua Dose Diária Definida (DDD)/mil habitantes/dia entre 2015 e 2017 se manteve entre 4,19 - 5,16. A quantidade dispensada sugere um consumo exagerado de antidepressivos nesse Município, sendo assim, é de suma importância ter uma regulação apropriada e uma prescrição racional dessas substâncias.

Palavras chave: Psicotrópicos, antidepressivos, dispensação, prescrição, farmacoepidemiologia.

\begin{abstract}
This is a retrospective study carried out in Capitão Leônidas Marques City, PR, with the goal of outlining dispensation profile of the antidepressants between January 2015 and December 2017 in the city. The data was obtained from quarterly psychotropic reports submitted for special control according to the law 344/98 of Heath Ministry of private and public systems, provided by sanitary surveillance. The antidepressants correspond to 55,3\% of psychotropic dispensed in this period and the most consumed was Amitriptilina 25mg, its Defined Daily Dose (DDD)/Thousand Inhabtants/Day between 2015 and 2017 remained among 4,19 - 5,16. The amount dispensed suggests an exaggerated consume of antidepressants in Capitão Leônidas Marques City, so appropriate regulation and a rational prescription of these drugs are of the utmost importance
\end{abstract}

Keywords: Psychotropics, antidepressants, dispensation, prescription, drug epidemiology.

\section{INTRODUÇÃO}

A depressão é uma doença relativamente comum pelo mundo e é diferente de alterações regulares de humor e respostas emocionais que atrapalham nos afazeres do dia-a-dia, podendo ter curta ou longa duração e ser classificada em leve, moderada ou grave (PAHO, 2017). 
Segundo a publicação "Depression and other common mental disorders: global health estimates" da World Health Organization - WHO (WHO, 2017), em 2015, havia cerca de 322 milhões de pessoas no mundo que viviam com depressão, um aumento de $18 \%$, se considerado o período de 2005 a 2015. No Brasil, estima-se que 5,8\% da população brasileira (11.548.577) sejam atingidas pela depressão (WHO, 2017).

A fisiopatologia da depressão é resultado de várias alterações no encéfalo tanto estruturais quanto metabólicas que causam alterações na produção de hormônios, principalmente alterações no eixo hipotálamo-hipófise-adrenal que está hiperativo em até metade dos pacientes. Hoje, o tratamento se baseia basicamente nos níveis de monoaminas, porém novos estudos vêm investigando mais as alterações póssinápticas que podem causar as alterações (SILVA, 2019; LAGE, 2010).

As classes de Antidepressivos usados no tratamento da depressão são os, Inibidores da Monoaminoxidase (IMAO), Tricíclicos, Inibidores Seletivos da Recaptação de Serotonina (ISRS), Inibidores Seletivos da Recaptação de Serotonina e Noradrenalina (ISRSN), Inibidores Seletivos da Recaptação de Dopamina (ISRD), entre outras (MORENO, MORENO, SOARES, 1999).

O uso abusivo dessas medicações podem gerar vários efeitos colaterais. Segundo Miller (2014) os comportamentos suicidas foram duas vezes mais comuns em pacientes que fizeram uso de doses acima do recomendado de antidepressivos, além de que jovens que já iniciem com terapêutica de altas doses tem risco elevado de provocarem autolesões.

De acordo com dados de acesso público, obtidos no Medical Expenditure Panel Survey (MEPS), o número de prescrições de fármacos psicotrópicos nos Estados Unidos cresceu 117\% entre 1999 e 2013. Já as mortes devido à overdose desses fármacos aumentaram 240\%, em 1999 eram 1.31 mortes por 100.000 habitantes e em 2013 esse número foi para 4.46 mortes por 100.000 habitantes.

\section{METODOLOGIA}

Trata-se de um estudo retrospectivo focado na dispensação de antidepressivos submetidos a controle especial. O primeiro passo, foi submeter o projeto para avaliação do Comitê de Ética em Pesquisa com Seres Humanos do Centro Universitário Assis Gurgacz (CEP-FAG), bem como conseguir a autorização da Secretaria Municipal de Saúde de Capitão Leônidas Marques, para acesso aos dados necessários. Após a aprovação do CEP-FAG (parecer no. 2.806.806), os pesquisadores iniciaram a coleta e obtenção de dados a partir dos RTs (relatórios trimestrais) em posse da vigilância sanitária do Município de Capitão Leônidas Marques - PR, no período de Janeiro de 2015 à Dezembro de 2017.

As informações relevantes a este estudo foram compiladas em tabela esquemática composta de data, medicamento dispensado e a tipologia do estabelecimento (público ou privado):

A quantificação do uso desses antidepressivos foi feita a partir da analise destes e o cálculo da Dose Diária Definida - DDD, conforme a fórmula abaixo, onde o CMA (mg) corresponde a quantidade em miligramas estimada para o consumo anual das substâncias:

DDD /mil habitantes/ dia=CMA(mg)x1000/(DDD x população x 365 dias)

Quanto à população, utilizou-se como base o censo do IBGE de 2010, no qual o total de habitantes era de 14.970, mas hoje a população é estimada em 15.740 habitantes (IBGE, 2018). 


\section{RESULTADOS E DISCUSSÃO}

Foram analisados e tabulados dados de 156 RTs fornecidas pela Vigilância Sanitária de Capitão Leônidas Marques correspondendo as 11 Farmácias do Município, Hospital e Sistema Municipal de Saúde, sendo 4 RTs por ano de cada estabelecimento. O Sistema Municipal de Saúde foi responsável por 98,12\% de toda a dispensação de antidepressivos.

Como observa-se, a Fluoxetina, em 2015, foi amplamente usada, chegando a um total de 110.336 unidades dispensadas, correspondendo a $35 \%$ de toda a dispensação de antidepressivos de 2015, porém nos anos seguintes sofreu uma queda na sua dispensação. Por outro lado o antidepressivo que mais aumentou sua porcentagem de dispensação entre 2015 e 2017 foi o Oxalato de Escitalopram, que em 2015 correspondia a 3,5\% de toda a dispensação de antidepressivos e em 2017 passou a representar $20,5 \%$ dessa dispensação, então teve um aumento significativo em apenas 2 anos. Nesse mesmo ano o Oxalato de Escitalopram, em relação a população de Capitão Leônidas Marques, teve sua DDD de 12,22/mil habitantes/dia, ultrapassando assim a DDD recomendada pela WHO que é de 10/mil habitantes/dia para esse fármaco. O valor total de antidepressivos no período pode ser observado no quadro 1.

Isso pode ser explicado pelo fato de a Fluoxetina pode causar mais disfunção sexual, ter maior chance de ser inibida pelo Citocromo P450 (CYP 450) e poder ser menos eficaz que uma intervenção psicoterapêutica em relação ao Oxalato de Escitalopram que hoje está na lista dos mais prescritos no mundo. Essa diferença de uso também se deve ao fato que, em alguns pacientes em farmacoterapia com fluoxetina, houve um aumento na sua ansiedade e agitação levando a uma necessidade de troca do fármaco e maior receio na sua prescrição. Além disso, hoje em dia não se ter mais a crença de que fluoxetina emagrece e sabe-se que 0 escitalopram apresenta menos efeitos colaterais (CORREIA, 2007).

Quadro 1. Total de antidepressivos dispensados entre 2015 a 2017

\begin{tabular}{|l|l|l|l|}
\hline & \multicolumn{1}{|c|}{$\mathbf{2 0 1 5}$} & \multicolumn{1}{|c|}{$\mathbf{2 0 1 6}$} & \multicolumn{1}{|c|}{$\mathbf{2 0 1 7}$} \\
\hline Total de Dispensação & 320.942 & 231.119 & 345.130 \\
\hline Dispensado pela Rede Pública & 317.680 & 227.974 & 341.515 \\
\hline Dispensado pela Rede Privada & 3.262 & 3.145 & 3.615 \\
\hline
\end{tabular}

Fonte: elaborado pelos autores

O total de Psicotrópicos dispensados no período foi de 1.619.879, desses os antidepressivos somam 897.262 unidades, o que corresponde a $55,3 \%$ dos psicotrópicos dispensados. A grande predominância na dispensação foi pela rede pública $(98,12 \%)$ e os fármacos mais dispensados foram Amitriptilina $25 \mathrm{mg}$ correspondendo a $41,05 \%$ do total de antidepressivos dispensados e em segundo lugar ficou a Fluoxetina com 32\%.

No quadro 2 podemos verificar a DDD calculada de cada antidepressivo dispensado pela rede pública de Capitão Leônidas Marques - PR em cada ano pesquisado. A Fluoxetina em 2015 teve uma DDD de 19,21/mil habitantes/dia, ficando muito próxima da DDD de 20/mil habitantes/dia recomendada pela WHO. Como já supracitado, o Oxalato de Escitalopram em 2017, teve uma grande dispensação, tendo uma DDD que ultrapassou a DDD recomendada pela WHO. 
Quadro 2. Dose Diária Definida em Capitão Leônidas Marques - PR de 2015-2017

\begin{tabular}{|l|c|c|c|}
\hline \multicolumn{1}{|c|}{ MEDICAMENTO } & $\begin{array}{c}\text { DDD } \\
\mathbf{2 0 1 5}\end{array}$ & $\begin{array}{c}\text { DDD } \\
\mathbf{2 0 1 6}\end{array}$ & $\begin{array}{c}\text { DDD } \\
\mathbf{2 0 1 7}\end{array}$ \\
\hline Amitriptilina 25mg & 4,19 & 4,67 & 5,16 \\
\hline Amitriptilina 75mg & 0,00 & 0,22 & 0,16 \\
\hline Bupropiona 150mg & 0,70 & 1,90 & 1,67 \\
\hline Citalopram 20mg & 4,00 & 0,12 & 0,00 \\
\hline Clomipramina 25mg & 0,36 & 0,33 & 0,51 \\
\hline Fluoxetina 20mg & 19,21 & 0,00 & 8,17 \\
\hline Fluoxetina 20mg/ml & 0,00 & 0,03 & 0,00 \\
\hline Imipramina 25mg & 0,61 & 0,61 & 0,43 \\
\hline Mirtazapina 45mg & 0,01 & 0,00 & 0,00 \\
\hline Nortriptilina 25mg & 0,29 & 0,93 & 1,27 \\
\hline Oxalato de Escitalopram 10mg & 1,89 & 5,90 & 12,22 \\
\hline Paroxetina 20mg & 4,15 & 4,35 & 4,07 \\
\hline Sertralina 50mg & 6,22 & 6,94 & 10,73 \\
\hline Trazodona 50mg & 0,00 & 0,00 & 0,00 \\
\hline Cloridrato de Venlafaxina 75mg & 0,82 & 0,04 & 0,00 \\
\hline Cloridrato de Venlafaxina 150mg & 0,81 & 0,04 & 0,00 \\
\hline
\end{tabular}

Fonte elaborada pelos autores

\section{CONSIDERAÇÕES FINAIS}

Amitriptilina foi o fármaco mais dispensado, o que mais aumentou a dispensação durante o período foi o Escitalopram e a fluoxetina mesmo sendo a segunda mais dispensada teve uma queda significativa na sua dispensação. O estudo busca resultados compatíveis com a realidade do Município, porém apesar da obrigatoriedade de do envio dos RTs para a vigilância sanitária, alguns RTs de alguns trimestres estavam faltando, então a amostra representa apenas estabelecimentos participantes, mas nelas contém os principais problemas referentes à prescrição e consumo de Antidepressivos em Capitão Leônidas Marques - PR. Outro ponto é que nem todos os fármacos dispensados são consumidos, então assim os dados podem ser superestimados. Mesmo com essas limitações identificadas, considera-se que os resultados sejam compatíveis com a realidade de consumo das substâncias presentes neste estudo.

Por meio dos resultados apresentados, mostra-se necessário o incentivo à prescrição médica racional de antidepressivos, bem como 0 uso da multidisciplinaridade para tratamento da depressão, através da associação de outras medidas terapêuticas, como a Terapia Cognitivo-Comportamental (TCC). Além disso, o envolvimento de outros profissionais da área da saúde é de extrema importância, como, por exemplo, os farmacêuticos que, segundo Santos, Valiatti \& Salvi (2017), atuam na dispensação dos fármacos, na farmacovigilância e nas orientações quanto ao uso dos medicamentos, o que pode promover benefícios ao usuário e uso o racional da medicação. Faz-se necessário ainda a realização de mais estudos nessa área para detectar prescrição indiscriminada, excesso de uso e possíveis problemas que essa população esteja sofrendo. 


\section{REFERÊNCIAS}

CORREIA, D.T.; GUERREIRO, D.F.; OLIVEIRA, S.; FIGUEIRA, M.L. Diferenças farmacodinâmicas e farmacocinéticas entre os SSRI implicações na prática e clínica. Acta Médica Portuguesa, v. 20, p. 167-74, 2007. Disponível em: $<$ http://www.gruponitro.com.br/atendimento-a-

profissionais/\%23/pdfs/artigos/farmacologia/farmacologia dos antidepressivos.pdf.> Acesso em: 29 out. 2018,

LAGE, J.T. Neurobiologia da Depressão. Repositório Aberto, abr 2010. Disponível em: $<$ https://repositorioaberto.up.pt/bitstream/10216/53466/2/Neurobiologia\%20da\%20Depresso.pdf> Acesso em 09 out. 2018.

MEPS. MEPS HC-033A: 1999 Prescribed Medicines. Medical Expenditure Panel Survey, ago 2002. Disponível em: $<$ https://meps.ahrq.gov/data stats/download data/pufs/h33a/h33adoc.pdf> Acesso em 09 de Outubro de 2018

MEPS. MEPS HC-160A: 2013 Prescribed Medicines. Medical Expenditure Panel Survey (MEPS), ago 2015.2 Disponível em: $<$ https://meps.ahrq.gov/data stats/download data/pufs/h160a/h160adoc.pdf>.

Acesso em: 09 out. 2018

MILLER, M.; SWANSON, S.A.; AZRAEL, D.; PATE, V.; STÜRMER, T. Jama Network, jun 2014. Antidepressant Dose, Age, and the Risk. Disponível em: $<$ https://jamanetwork.com/journals/jamainternalmedicine/fullarticle/1863925 $>$. Acesso em 09 out. 2018. doi:10.1001/jamainternmed.2014.1053

MORENO, R.A.; MORENO, D.H.; SOARES, M.B. Psicofarmacologia de antidepressivos. Revista Brasileira de Psiquiatria, v. 21, p. 24-40, mai 1999. http://dx.doi.org/10.1590/S1516-44461999000500006

PAHO. Aumenta o número de pessoas com depressão no mundo. Organização Pan-Americana da Saúde (PAHO), 2017. Disponível em: $<$ https://www.paho.org/bra/index.php?option=com content\&view=article\&id=5354:au menta-o-numero-de-pessoas-com-depressao-no-mundo\&ltemid=839>. Acesso em 09 out. 2018,

SANTOS, P.A.; VALIATTI, T.B.; SALVI, J.O. Perfil de Medicamentos Psicoativos dispensados na farmácia básica municipal de ji-Paraná, Rondônia. Revista Eletrônica de Farmácia, Goiânia/GO, v. 14, n. 3, p. 32-42, 2017. $<$ https://doi.org/10.5216/ref.v14i3.42711>. Acesso em 29 out. 2018.

SILVA, A. P., \& LINARTEVICHI, V. (2019). AVALIAÇÃO DA ORIGEM DAS PRESCRICOOES DE MEDICAMENTOS PSICOTRÓPICOS EM UM MUNICÍPIO DO OESTE DO PARANÁ. FAG JOURNAL OF HEALTH (FJH), 1(2), 150-153. https://doi.org/10.35984/fih.v1i2.100

WHO. ATC/DDD Index. World Health Organization Collaborating Centre for Drug Statistics Methodology, dez 2017. Disponível em: <https://www.whocc.no/atc ddd index/> Acesso em: 08 nov. 2018 